arXiv:0901.3818

\title{
Residual effects of heavy sparticles in the bottom quark Yukawa coupling: a comparative study for the MSSM and NMSSM
}

\author{
Wenyu Wang ${ }^{1}$, Zhaohua Xiong ${ }^{1}$, Jin Min Yang ${ }^{2}$ \\ 1 Institute of Theoretical Physics, College of Applied Science, \\ Beijing University of Technology, Beijing 100020, China \\ ${ }^{2}$ Key Laboratory of Frontiers in Theoretical Physics, Institute of Theoretical Physics, \\ Chinese Academy of Sciences, Beijing 100190, China
}

\begin{abstract}
If the sparticles are relatively heavy (a few TeV) while the Higgs sector is not so heavy $\left(m_{A}\right.$ is not so large), the Higgs boson Yukawa couplings can harbor sizable quantum effects of sparticles and these large residual effects may play a special role in probing supersymmetry at foreseeable colliders. In this work, focusing on the supersymmetric QCD effects in the $h b \bar{b}$ coupling ( $h$ is the lightest CP-even Higgs boson), we give a comparative study for the two popular supersymmetric models: the MSSM and NMSSM. While for both models the supersymmetric QCD can leave over large residual quantum effects in $h b \bar{b}$ coupling, the NMSSM can allow for a much broader region of such effects. Since these residual effects can be over $20 \%$ for the $h b \bar{b}$ coupling (and thus over $40 \%$ for the ratio $\left.\operatorname{Br}(h \rightarrow b \bar{b}) / \operatorname{Br}\left(h \rightarrow \tau^{+} \tau^{-}\right)\right)$, future measurements may unravel the effects of heavy sparticles or even distinguish the two models.
\end{abstract}

PACS numbers: 14.80.Cp,12.60.Fr,11.30.Qc

\section{INTRODUCTION}

Supersymmetry is a prime candidate for new physics beyond the Standard Model (SM). Among various supersymmetric models the most extensively studied is the minimal supersymmetric model (MSSM) [1]. Another popular supersymmetric model, which may be equally or more attractive compared with the MSSM, is the nextto-minimal supersymmetric model (NMSSM) 2] since it can solve the $\mu$-problem and alleviate the little hierarchy. These models will soon be put to the test at the LHC. Thus, their phenomenological study is important and urgent.

Although the most convincing evidence of supersymmetry is the detection of sparticle (sfermions, gauginos or Higgsinos) productions, the indirect probe through detecting the quantum effects of virtual sparticles in some measurable interactions will play a complementary role. If the sparticles are relatively heavy (say above a few $\mathrm{TeV}$ ) and hence cannot be directly detected at the LHC, the indirect probe through quantum effects could be important. For this end, the Higgs boson Yukawa interactions may play a special role since they can harbor sizable quantum effects of heavy sparticles when the Higgs sector is not so heavy (i.e. $m_{A}$ is not so large). The dominant quantum effects of sparticles are from the supersymmetric QCD interaction and in the literature the calculations have been performed in the MSSM for such supersymmetric QCD effects in the Higgs boson Yukawa couplings [3, 4] and the associated Higgs production processes at the LHC [5]. The studies in the decoupling limit with heavy sparticles showed that for a light Higgs sector $\left(m_{A}\right.$ is not large) the supersymmetric QCD can leave over large residual quantum effects in both the decay $h \rightarrow b \bar{b}[4]$ and the productions at the LHC [5]. Given the popularity of the NMSSM, it is necessary to extend the study to the NMSSM. This is the aim of this work.

In this work we will focus on the supersymmetric QCD effects in the $h b \bar{b}$ coupling ( $h$ is the lightest CP-even neutral Higgs boson) and perform a comparative study for the two popular supersymmetric models: the MSSM and NMSSM. Such a study is interesting for two points:

(i) The study of the NMSSM can accommodate the study of the MSSM, and in some limit the NMSSM results can reduce to the MSSM results. One can envisage that the supersymmetric residual effects in the MSSM can be magnified in the NMSSM. So the future measurements of such effects may be useful in telling the difference of the two models.

(ii) Compared with the MSSM, the NMSSM may predict a different tree-level $h b \bar{b}$ coupling and different loop contribution. In the NMSSM the mass matrix and mixings of the Higgs bosons are enriched and thus the components of the lightest CP-even Higgs boson $h$ are different from the MSSM values. The residual supersymmetric QCD effects in the NMSSM may be larger and more interesting.

Note that supersymmetry is a decoupling theory and all low-energy observables will recover their corresponding SM predictions when the mass scale of all supersymmetric particles (including the masses of sparticles and $m_{A}$ ) take their heavy limits. The large residual quantum effects of sparticles in the Higgs Yukawa couplings happen only in case that sparticles are heavy but $m_{A}$ is light. If 
both sparticles and $m_{A}$ take their heavy limit, the residual effects of supersymmetry do vanish. Since so far such a split scenario (with light Higgs bosons and relatively heavy sparticles) remains possible, we should check its phenomenological consequence.

\section{CALCULATIONS}

We start our analysis by recapitulating the basics of the NMSSM. In the NMSSM a singlet Higgs superfield $\hat{S}$ is introduced and the Higgs terms in the superpotential are given by

$$
\lambda \hat{S} \hat{H}_{d} \cdot \hat{H}_{u}-\frac{\kappa}{3} \hat{S}^{3},
$$

where $\hat{H}_{u}$ and $\hat{H}_{d}$ are the Higgs doublet superfields, and $\lambda$ and $\kappa$ are the dimensionless constants. Note that there is no explicit $\mu$-term and an effective $\mu$-parameter is generated when the scalar component $(S)$ of $\hat{S}$ develops a vev $s: \mu_{e f f}=\lambda s$. The corresponding soft SUSY breaking terms are given by

$$
-A_{\lambda} \lambda S H_{d} \cdot H_{u}-\frac{A_{\kappa}}{3} \kappa S^{3}+\text { h.c. } .
$$

So the scalar Higgs potential is given by

$$
\begin{aligned}
V_{F}= & \left|\lambda H_{d} \cdot H_{u}-\kappa S^{2}\right|^{2}+|\lambda S|^{2}\left(\left|H_{d}\right|^{2}+\left|H_{u}\right|^{2}\right), \\
V_{D}= & \frac{g_{2}^{2}}{2}\left(\left|H_{d}\right|^{2}\left|H_{u}\right|^{2}-\left|H_{d} \cdot H_{u}\right|^{2}\right) \\
& +\frac{g_{1}^{2}+g_{2}^{2}}{8}\left(\left|H_{d}\right|^{2}-\left|H_{u}\right|^{2}\right)^{2} \\
V_{\text {soft }}= & m_{d}^{2}\left|H_{d}\right|^{2}+m_{u}^{2}\left|H_{u}\right|^{2}+m_{s}^{2}|S|^{2} \\
& -\left(A_{\lambda} \lambda S H_{d} \cdot H_{u}+\frac{\kappa}{3} A_{\kappa} S^{3}+\text { h.c. }\right)
\end{aligned}
$$

where $g_{1}$ and $g_{2}$ are the coupling constant of $U_{Y}(1)$ and $S U_{L}(2)$, respectively. So we can see that in the limit of vanishing $\lambda, \kappa, A_{\kappa}$ and with the input of the effective $\mu_{e f f}$, the NMSSM can reduce to the MSSM.

With the vevs $v_{u}, v_{d}$ and $s$, the scalar fields are expanded as

$$
\begin{aligned}
H_{d} & =\left(\begin{array}{c}
v_{d}+\phi_{d}+i \varphi_{d} \\
H_{d}^{-}
\end{array}\right), \\
H_{u} & =\left(\begin{array}{c}
H_{u}^{+} \\
v_{u}+\phi_{u}+i \varphi_{u}
\end{array}\right), \\
S & =s+\sigma+i \xi .
\end{aligned}
$$

The mass eigenstates can be obtained by unitary rotations of interaction states, e.g., for the CP-even neutral mass eigenstates $\left(h, H_{1}, H_{2}\right)$ we have

$$
\left(h, H_{1}, H_{2}\right)^{T}=\sqrt{2} U^{H}\left(\phi_{d}, \phi_{u}, \sigma\right)^{T} .
$$

So the lightest CP-even neutral Higgs boson $h$ is composed by

$$
h=U_{11}^{H} \sqrt{2} \phi_{d}+U_{12}^{H} \sqrt{2} \phi_{u}+U_{13}^{H} \sqrt{2} \sigma .
$$

In the MSSM, $h$ can be decomposed in the same way except that without the singlet component, $U^{H}$ is a $2 \times 2$ matrix and can be parameterized in terms of a mixing angle $\alpha$.

The sbottom squared-mass matrix in the NMSSM is same as in the MSSM with $\mu$ replaced by $\mu_{e f f}$. In the basis of $\left(\tilde{b}_{L}, \tilde{b}_{R}\right)$, the squared-mass matrix is given by

$$
\mathcal{M}_{\tilde{b}}^{2}=\left(\begin{array}{cc}
M_{L}^{2} & m_{b} X_{b} \\
m_{b} X_{b} & M_{R}^{2}
\end{array}\right),
$$

where

$$
\begin{aligned}
X_{b} & =A_{b}+\mu_{e f f} \tan \beta, \\
M_{L}^{2} & =M_{\tilde{Q}}^{2}+m_{b}^{2}+M_{Z}^{2}\left(I_{3}^{b}-Q_{b} s_{W}^{2}\right) \cos 2 \beta, \\
M_{R}^{2} & =M_{\tilde{b}_{R}}^{2}+m_{b}^{2}+M_{Z}^{2} Q_{b} s_{W}^{2} \cos 2 \beta .
\end{aligned}
$$

with $s_{W} \equiv \sin \theta_{W}, \tan \beta=v_{u} / v_{d}, I_{3}^{b}$ and $Q_{b}$ being respectively the isospin and electric charge of the $b$-quark, $M_{\tilde{Q}}$ and $M_{\tilde{b}_{R}}$ being the soft breaking masses, and $A_{b}$ being the soft breaking trilinear coupling. The sbottom mass eigenstates $\left(\tilde{b}_{1}, \tilde{b}_{2}\right)$ are obtained by the unitary rotation of the interaction eigenstates:

$$
\left(\begin{array}{c}
\tilde{b}_{L} \\
\tilde{b}_{R}
\end{array}\right)=Z_{D}\left(\begin{array}{l}
\tilde{b}_{1} \\
\tilde{b}_{2}
\end{array}\right)=\left(\begin{array}{cc}
\cos \theta_{\tilde{b}} & -\sin \theta_{\tilde{b}} \\
\sin \theta_{\tilde{b}} & \cos \theta_{\tilde{b}}
\end{array}\right)\left(\begin{array}{l}
\tilde{b}_{1} \\
\tilde{b}_{2}
\end{array}\right)
$$

where the unitary matrix $Z_{D}$ parameterized by a mixing angle $\theta_{\tilde{b}}$ diagonalizes the mass matrix in Eq.(11).

The coupling of sbottoms with the singlet component $\sigma$ of the Higgs boson $h$ comes from the F-term of the superpotential, given by

$$
-\frac{g_{2} m_{b}}{2 m_{W} \cos \beta} \lambda v_{u} \sqrt{2} \sigma \tilde{b}_{L}^{*} \tilde{b}_{R}+\text { h.c. . }
$$

In terms of the mass eigenstates of sbottoms, the vertex $h \tilde{b}_{i} \tilde{b}_{j}$ takes the form

$$
V_{h \tilde{b}_{i} \tilde{b}_{j}}=-i \frac{g_{2} m_{b}}{2 m_{W} \cos \beta}\left(U_{11}^{H} c_{d}^{i j}+U_{12}^{H} c_{u}^{i j}+U_{13}^{H} c_{s}^{i j}\right)
$$

where $c_{s}^{i j}$ is only for the NMSSM (or equivalently set $U_{13}^{H}=0$ for the MSSM), while $c_{d}^{i j}$ and $c_{u}^{i j}$ are present for both models. They are given by

$$
\begin{aligned}
c_{d}^{i j}= & {\left[2 m_{b}-\frac{m_{Z}^{2} \cos ^{2} \beta}{m_{b}}\left(1-\frac{2}{3} s_{W}^{2}\right)\right] Z_{D}^{1 i} Z_{D}^{1 j} } \\
& +\left[2 m_{b}-\frac{2 m_{Z}^{2} s_{W}^{2} \cos ^{2} \beta}{3 m_{b}}\right] Z_{D}^{2 i} Z_{D}^{2 j} \\
& +A_{b}\left(Z_{D}^{1 i} Z_{D}^{2 j}+Z_{D}^{2 i} Z_{D}^{1 j}\right), \\
c_{u}^{i j}= & \frac{m_{Z}^{2} \sin 2 \beta}{2 m_{b}}\left(1-\frac{2}{3} s_{W}^{2}\right) Z_{D}^{1 i} Z_{D}^{1 j} \\
& +\frac{m_{Z}^{2} s_{W}^{2} \sin 2 \beta}{3 m_{b}} Z_{D}^{2 i} Z_{D}^{2 j} \\
& +\mu_{e f f}\left(Z_{D}^{1 i} Z_{D}^{2 j}+Z_{D}^{2 i} Z_{D}^{1 j}\right), \\
c_{s}^{i j}= & \frac{\sqrt{2} \lambda m_{W} \sin \beta}{g_{2}}\left(Z_{D}^{1 i} Z_{D}^{2 j}+Z_{D}^{2 i} Z_{D}^{1 j}\right) .
\end{aligned}
$$


Now we calculate the SUSY QCD corrections to the vertex $h b \bar{b}$. At tree level it takes the same form in both the MSSM and NMSSM, given by

$$
V_{h b \bar{b}}^{0}=-i \frac{g_{2} m_{b} U_{11}^{H}}{2 m_{W} \cos \beta}
$$

The one-loop SUSY QCD corrections come from the Feynman diagrams shown in Fig 1 where we do not show the self-energy loop of $\bar{b}$. In our calculations we use the

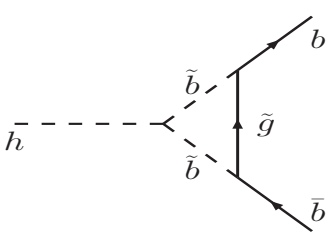

(a)

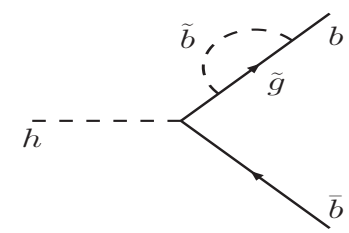

(b)
FIG. 1: Feynman diagrams for the SUSY QCD corrections to the vertex $h b \bar{b}$.

on-shell renormalization scheme 7] and take the external $b$ and $\bar{b}$ quarks on shell to get the effective vertex. With the corrections the effective vertex takes the form

$$
V_{h b \bar{b}}=V_{h b \bar{b}}^{0}\left(1+\Delta^{\mathrm{v}}+\Delta^{\mathrm{ct}}\right),
$$

where $\Delta^{\mathrm{v}}$ denotes the vertex correction from Fig 1 (a) and $\Delta^{\mathrm{ct}}$ is the counter term from the renormalization of $m_{b}$ and the wave functions of $b$ and $\bar{b}$. They are given by

$$
\begin{aligned}
\Delta^{\mathrm{v}}= & \frac{\alpha_{s}}{3 \pi}\left(c_{d}^{i j}+\frac{U_{12}^{H}}{U_{11}^{H}} c_{u}^{i j}+\frac{U_{13}^{H}}{U_{11}^{H}} c_{s}^{i j}\right) \\
& \times\left[m_{b}\left(Z_{D}^{1 i} Z_{D}^{1 j}+Z_{D}^{2 i} Z_{D}^{2 j}\right) C_{11}+m_{\tilde{g}}\left(Z_{D}^{1 i} Z_{D}^{2 j}\right.\right. \\
& \left.\left.+Z_{D}^{2 i} Z_{D}^{1 j}\right) C_{0}\right]\left(m_{b}^{2}, m_{h}^{2}, m_{b}^{2}, m_{\tilde{g}}^{2}, m_{\tilde{b}_{i}}^{2}, m_{\tilde{b}_{j}}^{2}\right), \\
\Delta^{\mathrm{ct}}= & -\frac{\alpha_{s}}{3 \pi}\left\{\frac { m _ { \tilde { g } } } { m _ { b } } \operatorname { s i n } ( 2 \theta _ { \tilde { b } } ) \left[B_{0}\left(m_{b}^{2}, m_{\tilde{g}}^{2}, m_{\tilde{b}_{1}}^{2}\right)\right.\right. \\
& \left.-B_{0}\left(m_{b}^{2}, m_{\tilde{g}}^{2}, m_{\tilde{b}_{2}}^{2}\right)\right]-2 m_{b}^{2}\left[B_{1}^{\prime}\left(m_{b}^{2}, m_{\tilde{g}}^{2}, m_{\tilde{b}_{1}}^{2}\right)\right. \\
& \left.+B_{1}^{\prime}\left(m_{b}^{2}, m_{\tilde{g}}^{2}, m_{\tilde{b}_{2}}^{2}\right)\right]-2 m_{b} m_{\tilde{g}} \sin \left(2 \theta_{\tilde{b}}\right) \\
& \left.\times\left[B_{0}^{\prime}\left(m_{b}^{2}, m_{\tilde{g}}^{2}, m_{\tilde{b}_{1}}^{2}\right)-B_{0}^{\prime}\left(m_{b}^{2}, m_{\tilde{g}}^{2}, m_{\tilde{b}_{2}}^{2}\right)\right]\right\},
\end{aligned}
$$

where $\alpha_{s}$ is the strong coupling constant, $m_{\tilde{g}}$ is the gluino mass, and $B_{0,1}, B_{0,1}^{\prime}$ and $C_{0,11}$ are the scalar loop functions [8] which can be calculated by using LoopTools [9].

\section{NUMERICAL RESULTS}

In our calculation we use the package NMSSMTools [10] for the mass spectrum and the rotation matrix of the Higgs fields (to get the corresponding MSSM results we take the limit of very small values for $\lambda, \kappa$ and $A_{\kappa}$, and results are checked by using the package FeynHiggs [11]). To study the decoupling limit of SUSY particles, we assume all the soft breaking mass parameters
$\left(M_{\tilde{Q}}, M_{\tilde{b}_{R}}, m_{\tilde{g}}, A_{b}\right)$ and the parameter $\mu_{\text {eff }}$ are degenerate, which are collectively denoted by $M_{S U S Y}$. Then in the MSSM the SUSY parameters are $\left(M_{S U S Y}, M_{A}\right.$, $\tan \beta)$, while in the NMSSM there are three additional parameters $\left(\lambda, \kappa, A_{\kappa}\right)$. In our calculations we scan over these three additional parameters in the ranges

$$
-0.5<\lambda, \kappa<0.5, \quad-500 \mathrm{GeV}<A_{\kappa}<500 \mathrm{GeV}
$$

Note here we did not consider a large $\lambda$ or $\kappa$. Theoretically, the requirement of perturbativity up to some cut-off scale will set upper bounds on $\lambda$ and $\kappa$ at weak scale (if the cut-off scale is chosen to be the GUT scale, a stringent bound $\lambda^{2}+\kappa^{2} \lesssim 0.5$ is obtained [6]). Phenomenologically, a large $\lambda$ or $\kappa$ will incur stringent constraints from current experiments (see the last reference in [2]).

Before displaying the numerical results, we make some clarifications regarding to our numerical calculations:

(1) In our calculations we used the package NMSSMTools [10] which considered the loop corrections (especially the stop/sbottom loops) to the effective potential, the masses and mixing angles of the Higgs bosons. These corrections are important and cannot be ignored.

(2) Since we used the package NMSSMTools in which the Higgs mass matrices are diagonalized numerically, we did not use any approximate unitary transformation for our calculation.

(3) For the b-quark mass $m_{b}$, in the package NMSSMTools (and thus in our calculation), it is taken as the running mass $m_{b}(Q)$ (we take $Q=M_{S U S Y}$ ), which means that the sizable QCD loop effects are taken into account. Actually, although both the tree-level coupling $V_{h b \bar{b}}^{0}$ and the one-loop SUSY QCD contributions $\delta V_{h b \bar{b}}$ are proportional to $m_{b}$ and thus very sensitive to the value of $m_{b}$, the relative correction effects $\delta V_{h b \bar{b}} / V_{h b \bar{b}}^{0}$ (displayed in our numerical results) are not so sensitive to the value of $m_{b}$.

Now we present some numerical results. To see the general feature of the corrections, we first switch off the experimental constraints on the parameter space and in Figs 2 and 3 display the resluts of the relative correction effects

$$
\Delta_{S Q C D} \equiv \frac{\delta V_{h b \bar{b}}}{V_{h b \bar{b}}^{0}}=\Delta^{\mathrm{v}}+\Delta^{\mathrm{ct}} .
$$

We see that for a light $m_{A}$ the SUSY QCD with a large $M_{S U S Y}$ can leave over sizable effects in the coupling $h b \bar{b}$. But as $m_{A}$ gets heavy, such residual effects of SUSY QCD become small in magnitude, showing the decoupling behavior of supersymmetry. Compared with the MSSM results, the effects in the NMSSM can vary in a much broader region. While the corrections are always negative in the MSSM, in the NMSSM the corrections can be both negative and positive. 


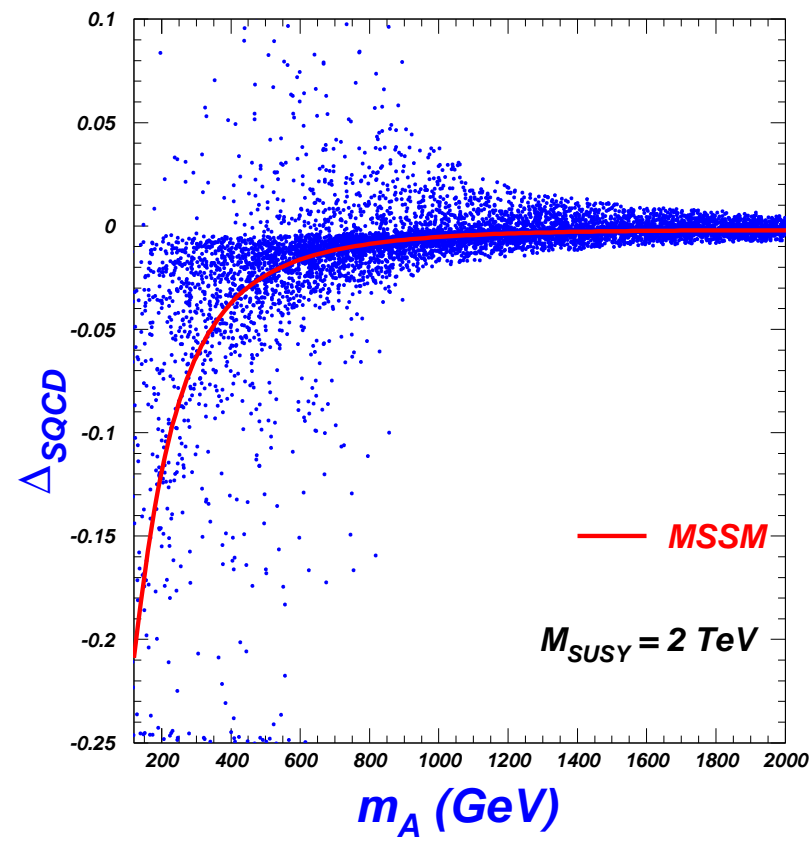

FIG. 2: SUSY QCD corrections to $h b \bar{b}$ coupling versus $m_{A}$ in the MSSM and NMSSM for $\tan \beta=20$. The scatter plots are for the NMSSM while the curves for the MSSM.
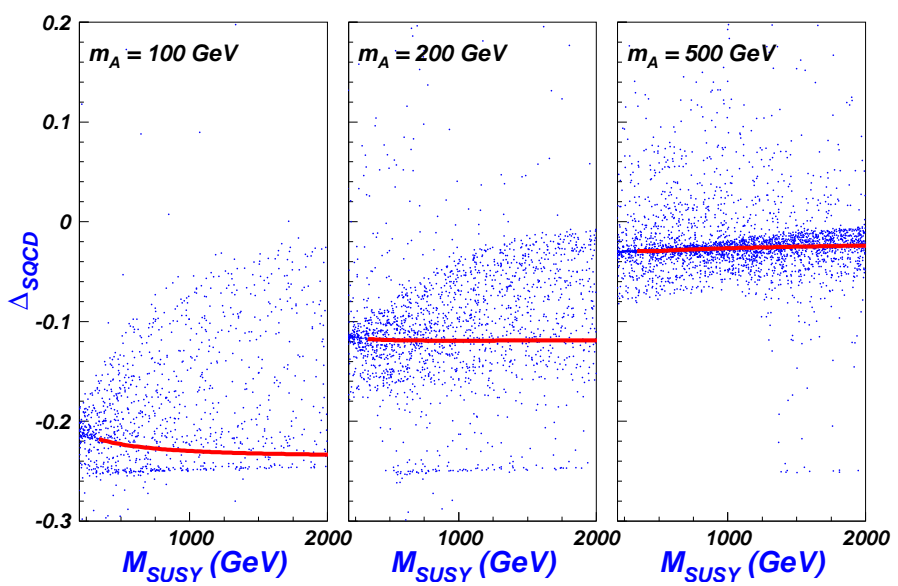

FIG. 3: Same as Fig. 2, but versus $M_{S U S Y}$ for fixed values of $m_{A}$.

In order to figure out in what areas of the NMSSM parameter space the one-loop SUSY QCD effects on the $h b \bar{b}$ coupling are sizable, we present a set of sample points in Table [1] We see that for the SUSY QCD loop effects on the $h b \bar{b}$ coupling to be sizable in the NMSSM, the ratio $U_{12}^{H} / U_{11}^{H}\left(U_{12}^{H}\right.$ and $U_{11}^{H}$ are respectively the components of $\phi_{u}$ and $\phi_{d}$ in $h$, as defined in Eq(10) plays the key role for the following reasons. As shown in Eq.(24), $\Delta_{S Q C D}$ is composed of two parts: the counter-term part $\Delta^{\mathrm{ct}}$ and the vertex-loop part $\Delta^{\mathrm{v}}$. We found that in most of the parameter space allowed by the LEP constraints, $\Delta^{\text {ct }}$ is negative and dominant in size, which is independent of how $h$ is composed of. Whereas, $\Delta^{\mathrm{v}}$ is positive and cancel $\Delta^{\text {ct }}$ to some extent. The size of $\Delta^{\mathrm{v}}$ can be enhanced by the ratio $U_{12}^{H} / U_{11}^{H}$, as shown in Eq.(21). Although the
TABLE I: Some sample points in the parameter space of the NMSSM, in which the SUSY QCD loop effects $\Delta_{S Q C D}$ can be of very different size. The mass parameters are in unit of $\mathrm{GeV}$. Here we fixed $M_{A}=200 \mathrm{GeV}$ and $\tan \beta=20$. With such fixed parameters and $M_{S U S Y}$ varying from $400 \mathrm{GeV}$ to $2 \mathrm{TeV}$, the value of $m_{h}$ in the MSSM varies from $106 \mathrm{GeV}$ to $119 \mathrm{GeV}$ while the corresponding values of $\Delta_{S Q C D}$ in the MSSM are about $-11 \%$.

\begin{tabular}{rrrrrrrr}
\hline \hline \multicolumn{1}{c}{$\lambda$} & $\kappa$ & $A_{\kappa}$ & $M_{S U S Y}$ & $U_{12}^{H} / U_{11}^{H}$ & $U_{13}^{H} / U_{11}^{H}$ & $m_{h}$ & $\begin{array}{c}\Delta_{S Q C D} \\
(\%)\end{array}$ \\
\hline 0.106 & -0.215 & 446 & 1788 & 16.2 & -0.023 & 117.4 & -4.1 \\
-0.116 & 0.180 & 333 & 1575 & 14.7 & 0.042 & 116.3 & -6.0 \\
0.133 & -0.256 & 393 & 1145 & 14.8 & -0.046 & 114.7 & -6.1 \\
0.123 & -0.164 & 460 & 1568 & 14.1 & -0.062 & 116.1 & -6.8 \\
-0.103 & -0.072 & -294 & 1544 & 10.1 & 0.118 & 114.8 & -12.0 \\
0.107 & 0.073 & -175 & 1831 & 9.7 & -0.100 & 115.2 & -12.4 \\
0.126 & 0.101 & -426 & 1738 & 7.3 & -0.069 & 114.8 & -15.5 \\
-0.106 & -0.123 & -377 & 1401 & 5.4 & 0.024 & 115.3 & -18.0 \\
0.110 & 0.130 & -324 & 1419 & 4.1 & -0.017 & 114.9 & -19.7 \\
0.106 & 0.406 & -304 & 623 & 0.5 & 0.002 & 99.4 & -24.5 \\
\hline \hline
\end{tabular}

ratio $U_{13}^{H} / U_{11}^{H}$ can also enhance the size of $\Delta^{\mathrm{v}}$, its effect is suppressed by the smallness of $U_{13}^{H}$ and $\lambda$ in $c_{s}^{i j}$ (in our scan we found that both $\lambda$ and $U_{13}^{H}$ are small in order to satisfy the LEP constraints encoded in the NMSSMTools). Therefore, as $U_{12}^{H} / U_{11}^{H}$ gets large, $\Delta^{\mathrm{v}}$ becomes more sizable and, due to its cancellation effect, the total correction effects become less sizable. Note that although the value of $m_{h}$ is below $114 \mathrm{GeV}$ for some points, these points can still satisfy the LEP constraints encoded in the NMSSMTools because the LEP bound on the MSSM $m_{h}$ is $92 \mathrm{GeV}$ (see Fig.2 in the paper by Barger et. al. in Ref.[2]). By the way, some comprehensive studies on the phenomenology of the NMSSM Higgs sector (which may be quite different from the phenomenology of the MSSM Higgs sector) have been performed in Ref. 2], where all the Higgs couplings and all the decay modes as well as productions have been intensively studied.

From Table 1 we see that although $\lambda$ is scanned in the range of $-0.5 \sim 0.5$, only small values of $\lambda(\sim 0.1$ in magnitude) survived the LEP constraints encoded in the NMSSMTools. As $\lambda$ gets large, the mixing between singlet and doublet Higgs fields becomes large (although the mixing is not totally determined by $\lambda$ and other parameters are also contributing) and, consequently, is more constrained by LEP experiments [2]. Such a small $\lambda$, together with other NMSSM parameters appearing in the mass matrix of the Higgs fields, leads to a small mixing between singlet and doublet Higgs fields. As shown in Table [. the singlet component $U_{13}^{H}$ in $h$ is small, which means that the lightest Higgs boson $h$ is not the singletlike one and instead it is doublet-dominant (MSSM-like). For the $h b \bar{b}$ coupling with such a MSSM-like $h$, the NMSSM can still allow for quite different SUSY QCD effects compared with the MSSM predictions. The reason is that, as discussed above, the SUSY QCD effects are sensitive to the ratio $U_{12}^{H} / U_{11}^{H}$, which is different in 
these two models (even for a small $\lambda$, the NMSSM can still allow for different-size components $U_{12}^{H}$ and $U_{11}^{H}$ in $h$ because other NMSSM parameters are also contributing to the mass matrix and mixings of the Higgs fields).

When we switch on the comprehensive experimental constraints encoded in the NMSSMTools [10, we obtain the results of $\Delta_{S Q C D}$ displayed in Fig,4. These experimental constraints are comprehensive, including the LEP II searches for the Higgs boson, various B-decays and the muon anomalous magnetic moment (muon $g-2$ ).

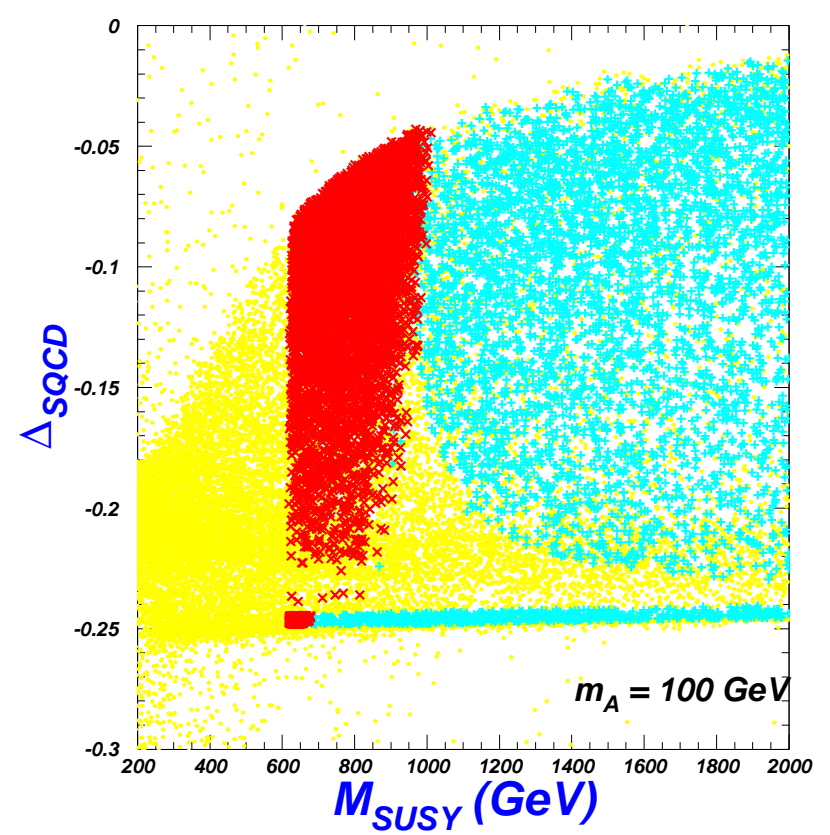

FIG. 4: Same as Fig. 3, but for $m_{A}=100 \mathrm{GeV}$ in the NMSSM. The dark-shaded region denoted by ' $x$ ' (red in color) are allowed by the experimental constraints considered in the NMSSMTools [10]. The light-shaded region denoted by '•' (yellow in color) are excluded by LEP experiment or B physics, and the medium-shaded region denoted by '+' (skyblue in color) are excluded by the muon $g-2$.

From Fig 4 we see that in the special scenario under our consideration (all soft breaking mass parameters are degenerate), $M_{S U S Y}$ is constrained in a certain range. The range of $M_{S U S Y} \lesssim 600 \mathrm{GeV}$ is not allowed by LEP experiments, while $M_{S U S Y} \gtrsim 1 \mathrm{TeV}$ cannot explain the muon $g-2$ data (we require the supersymmetric effects to account for the deviation of $a_{\mu}^{e x p}-a_{\mu}^{S M}=(29.5 \pm$ $8.8) \times 10^{-10}$ at $2 \sigma$ level). In the allowed region of the parameter space, the SUSY QCD corrections to the $h b \bar{b}$ coupling can still be significant, over $20 \%$ in magnitude. If we switch off the muon $g-2$ constraint ( the hadronic contribution to $a_{\mu}^{S M}$ is not so certain [12]), the allowed parameter space gets much broader, as shown in Fig 4. In our study we did not require supersymmetry to explain various plausible evidences of dark matter.

Note that the SUSY contributions to the muon $g-2$ are sensitive to the soft masses in slepton sector and chargino sector, but not dependent on the squark or gluino mass involved in our SUSY QCD loops. The stringent con- straint from the muon $g-2$ data shown in Fig 4 comes from our simple assumption that all soft masses (in the squark sector, the slepton sector and the gaugino sector) are degenerate. Of course, the muon $g-2$ constraint is not necessary; without such an assumption of degeneracy, the constraint is lifted.

Since the SUSY QCD residual effects can be over $20 \%$ for the $h b \bar{b}$ coupling and thus over $40 \%$ for the ratio of the branching fractions (SUSY QCD does not contribute to $h \rightarrow \tau^{+} \tau^{-}$at one-loop level)

$$
R_{b / \tau}=\frac{B r(h \rightarrow b \bar{b})}{B r\left(h \rightarrow \tau^{+} \tau^{-}\right)},
$$

future measurements at the LHC or ILC may unravel such supersymmetric effects. This ratio $R_{b / \tau}$ is proposed in [13] as a probe to new physics. To measure this ratio at the LHC, one may count the event numbers of the production of $h b \bar{b}$ followed respectively by the decay $h \rightarrow b \bar{b}$ and $h \rightarrow \tau^{+} \tau^{-}$, and the ratio of these two event-numbers can be a measure of $R_{b / \tau}$ (the difference of efficiency for $b$-tagging and $\tau$-tagging should be taken into account).

Finally, we make some remarks regarding to our results:

(1) We only investigated the SUSY QCD corrections, which is $\mathcal{O}\left(\alpha_{s}\right)$ and thus should be the most important among the SUSY corrections. Among the SUSY electroweak corrections, the Higgs-top Yukawa corrections, which are $\mathcal{O}\left(\alpha \frac{m_{t}^{2}}{m_{W}^{2}}\right)$, may also be sizable although they are seemingly not as large as the $\mathcal{O}\left(\alpha_{s}\right)$ SUSY QCD corrections. With the consideration of the SUSY electroweak corrections, our conclusion will remain qualatively unchanged.

(2) Since our main interest is the residual effects of heavy sparticles, we assumed all soft SUSYbreaking mass parameters are equal to $M_{S U S Y}$. This is a very strong simplifying assumption. If we lift such an assumption and consider the multiple free soft parameters, we will obtain the results which numerically are different to some extent while qualitatively exhibit the same feature, i.e., the NMSSM allows broader corrections than the MSSM, because the NMSSM parameters can complicate the mass matrix and mixings of Higgs fields.

(3) We should stress again that supersymmetry is a decoupling theory and the large residual quantum effects of sparticles in the Higgs Yukawa couplings are present only in case of a light $m_{A}$. If both $M_{S U S Y}$ and $m_{A}$ take their heavy limit, the SUSY effects will vanish. Such a decoupling behavior is similar in the NMSSM and MSSM, as shown in Fig.2. 


\section{SUMMARY}

We focused on the SUSY QCD effects in the $h b \bar{b}$ coupling and performed a comparative study for the two popular SUSY models: the MSSM and NMSSM. We found that for both models the SUSY QCD can leave over large residual quantum effects in $h b \bar{b}$ coupling if the sparticles are relatively heavy (a few $\mathrm{TeV}$ ) while the Higgs sector is not so heavy ( $m_{A}$ is not so large). Compared with the MSSM results, the NMSSM can allow for a much broader region of such residual effects. Since these residual effects can be over $20 \%$ in magnitude, fu- ture measurements of the $h b \bar{b}$ coupling may unravel such supersymmetric effects or even distinguish the two models.

\section{Acknowledgment}

This work was supported in part by the National Natural Science Foundation of China under grant Nos. 10821504, 10725526 and 10635030, and by the Natural Science Foundation of Beijing under grant No. 1072001.
[1] For a review, see, e.g., H. E. Haber, G. L. Kane, Phys. Rept. 117, 75 (1985).

[2] See, e.g., J. R. Ellis, et. al., Phys. Rev. D 39, 844 (1989); M. Drees, Int. J. Mod. Phys. A 4, 3635 (1989). S. F. King, P. L. White, Phys. Rev. D 52, 4183 (1995); B. Ananthanarayan, P.N. Pandita, Phys. Lett. B 353, 70 (1995); Phys. Lett. B 371, 245 (1996); Int. J. Mod. Phys. A12, 2321 (1997); B. A. Dobrescu, K. T. Matchev, JHEP 0009, 031 (2000); V. Barger, P. Langacker, H.-S. Lee, G. Shaughnessy, Phys. Rev. D 73,(2006) 115010; R. Dermisek, J. F. Gunion, Phys. Rev. Lett. 95, 041801 (2005); G. Hiller, Phys. Rev. D 70, 034018 (2004); F. Domingo, U. Ellwanger, JHEP 0712, 090 (2007); Z. Heng, et al., Phys. Rev. D 77, 095012 (2008); R. N. Hodgkinson, A. Pilaftsis, Phys. Rev. D 76, 015007 (2007); Phys. Rev. D 78, 075004 (2008); J. Cao,, H. E. Logan, J. M. Yang, arXiv:0901.1437 [hep-ph]; J. Cao, J. M. Yang, JHEP 0812, 006 (2008); Phys. Rev. D 78, 115001 (2008);

[3] C. S. Li, J. M. Yang, Phys. Lett. B 315, 367 (1993); A. Dabelstein, Nucl. Phys. B 456, 25 (1995); H. Eberl, et al., Phys. Rev. D 62, 055006 (2000); M. Carena, D. Garcia, U. Nierste, C. E. Wagner,Nucl. Phys. B 577, 88 (2000);
[4] H. E. Haber, et al., Phys. Rev. D 63, 055004 (2001);

[5] J. Cao, G. Gao, R. J. Oakes, J. M. Yang, Phys. Rev. D 68, 075012 (2003); G. Gao, et al., Phys. Rev. D 66, 015007 (2002); Phys. Rev. D 71, 095005 (2005).

[6] D. J. Miller, R. Nevzorov, P. M. Zerwas, Nucl. Phys. B 681, 3 (2004).

[7] A. Denner, Fortschr. Phys. 41 (1993)4

[8] G. Passarino and M. J. G. Veltman, Nucl. Phys. B 160, 151 (1979); G. 't Hooft and M. J. G. Veltman, Nucl. Phys. B 153, 365 (1979).

[9] T. Hahn, M. Perez-Victoria, Comput. Phys. Commun. 118, 153 (1999); T. Hahn, Nucl. Phys. Proc. Suppl. 135, 333 (2004).

[10] U. Ellwanger, J. F. Gunion, C. Hugonie, JHEP 0502, 066 (2005).

[11] M. Frank, et al., JHEP 0702, 047 (2007).

[12] J. P. Miller, et. al., Rept. Prog. Phys. 70, 795 (2007).

[13] J. Guasch, W. Hollik, S. Penaranda, Phys. Lett. B 515, 367 (2001); T. Ibrahim, P. Nath, Phys. Rev. D 68, 015008 (2003). 